\title{
GABAergic Neurons in the Mouse Superficial Dorsal Horn with Special Emphasis on their Relation to Primary Afferent Central Terminals
}

\author{
Akio Hiura, Hiroshi Ishizuka and Elizabeth López Villalobos* \\ Department of Oral Anatomy, Tokushima University School of Dentistry, Tokushima, Japan
}

Received December 25, 1990

Summary. Immunocytochemical studies were carried out on the morphological relation between primary afferent central terminals (C-terminals) and GABAergic neurons in the mouse superficial dorsal horn. The superficial dorsal horn is composed of many synaptic glomeruli comprising two types: Type I with centrally located CI-terminals surrounded by several dendrites and few axonal endings, and Type II with centrally located CII-terminals surrounded by several dendrites and a few axonal endings. The CI-terminals are sinuous or scalloped with densely packed agranular synaptic vesicles, a few granular synaptic vesicles and mitochondria, and show an electron dense axoplasm, whereas the CII-terminals are large and round or rectangular with evenly distributed agranular synaptic vesicles, a number of granular synaptic vesicles and mitochondria, and show an electron opaque axoplasm.

The immunoreaction of GABA was remarkable in the superficial laminae of the dorsal horn. Many interneuronal somata in the substantia gelatinosa showed GABAergic immunoreactivity. The immunoreaction was seen in the entire GABAergic neuroplasm, but not in the nucleus and its envelope. Most GABAergic features appeared as dendrites making postsynaptic contact with CI- or CII-terminals; i.e., numerous C-terminals made presynaptic contact with GABAergic dendrites. GABA immunoreactivity was seen over round synaptic vesicles and mitochondrial membranes. A few CIIterminals made presynaptic contact with GABAergic interneuronal somata. Previous physiological and anatomical studies have suggested that not only the cutaneous nociceptive primary afferent $\mathrm{C}$-terminals but also mechanoreceptive primary afferent $\mathrm{C}$-terminals make presynaptic contact with the GABAergic dendrites, boutons and soma. The presynaptic relation of these primary afferents with GABAergic neurons seems to provide morphological support for the essential feature of the gate control theory: primary afferent fibers may play a part in the modulation of nociceptive information via GABAergic neurons in the superficial dorsal horn. Small GABAergic terminals were found to make contact with blood capillaries suggesting the release of GABA into circulation.

The superficial dorsal horn is important for the modulation of pain transmission from the periphery (CERvero, 1986, 1989). GABA ( $\boldsymbol{\gamma}$-aminobutyric acid) is known as a presynaptic inhibitory transmitter (ECCLES et al., 1963; LEVY, 1977). Accordingly in the last ten years many studies have focused on the immunocytochemical identification of GABAergic neurons in the spinal dorsal horn. The results of most studies are consistent with the conclusion that the substantia gelatinosa is rich in GABAergic boutons and that these are presynaptic to the primary afferent central terminals (C-terminals) conveying nociceptive impulses.

The C-terminals in the superficial dorsal horn are classified into two types: CI-terminals, which are considered to be central terminals of unmyelinated afferent fibers, and CII-terminals, considered to be fine myelinated afferents (RIBEIRO-DA-SILVA and COIMBRA, 1982). We demonstrated the same types of C-terminals in the mouse superficial dorsal horn (HiURA et al., 1990). Recently, there have been a few reports on the morphological relationship between GABAergic neurons and C-terminals. TODD and LOCHHEAD (1990) observed synaptic contact between

\footnotetext{
*Present address of E. L. V.: Department of Anatomy, Veracruz University School of Medicine, AV, Atenas y Managua S/N, Minatitlan, Veracruz, Mexico
} 
GABAergic terminals and CI-terminals by immunogold labeling. CARLTON and HAYES (1990) clearly demonstrated many GABAergic dendrites making postsynaptic contact with C-terminals in the monkey spinal dorsal horn. KADURI et al. (1987) identified GABAergic neurons in the mouse spinal cord using an antibody to GABA, but did not refer to the synaptic relationship between GABAergic boutons and C-terminals in the superficial laminae (I-III) of the dorsal horn.

In this study, we examined the GABAergic neurons in the mouse superficial dorsal horn with special interest given to the anatomical relationship between GABAergic dendrites or boutons and the synaptic glomeruli with C-terminals.

\section{MATERIALS AND METHODS}

Adult DDY mice under Nembutal anesthesia were perfused through the left ventricle with saline followed by fixative of $1 \%$ paraformaldehyde and $0.5 \%$ glutaraldehyde in $0.1 \mathrm{M}$ phosphate buffered saline (PBS, pH 7.4). The lumbar spinal cord was then

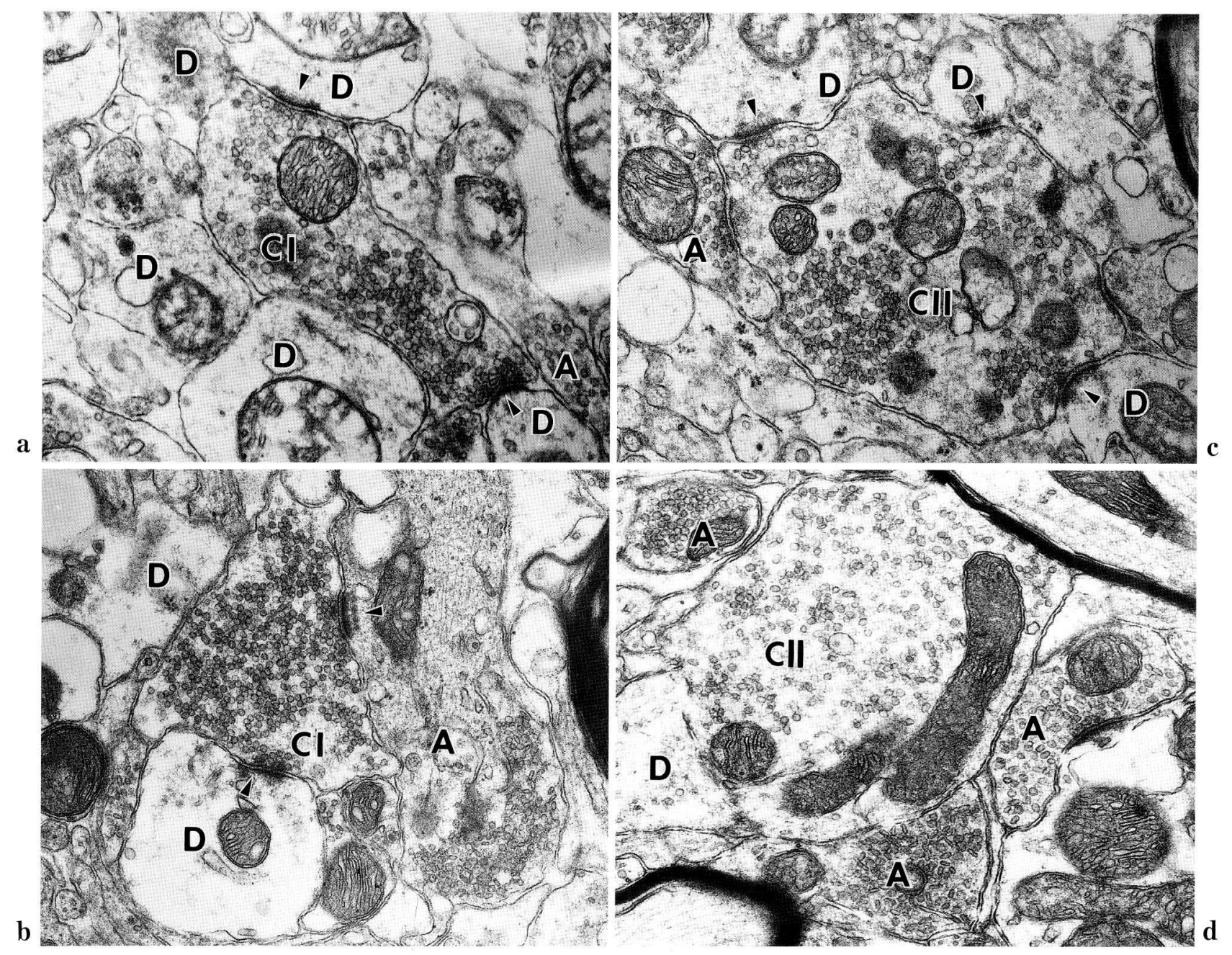

Fig. 1. Type I and Type II synaptic glomeruli in the normal superficial dorsal horn. a. A typical sinuous CI-terminal $(C I)$ making presynaptic contact with dendrites $(D)$ and symmetrical contact with a small axon terminal $(A)$. b. A CI-terminal $(C I)$ with closely packed round synaptic vesicles making presynaptic contact with a dendrite $(D)$ and an axon terminal $(A)$ containing many discoid synaptic vesicles. $\mathbf{c}$. A large CII-terminal with many round synaptic vesicles and mitochondria, making presynaptic contact with dendrites $(D)$ or symmetrical contact with a small axon terminal $(A)$. d. A large CII-terminal $(C I I)$ with round, evenly distributed synaptic vesicles, making symmetrical contacts with axon terminals $(A)$ including many discoid synaptic vesicles. Arrowheads indicate thick postsynaptic membranes. a, c, d: $\times 27,000, b: \times 24,000$ 
excised and immersed in the same fixative for $1 \mathrm{~h}$ at $4^{\circ} \mathrm{C}$. Sections of $50 \mu \mathrm{m}$ were cut in a Vibratome (Lancer) and stored in the buffer. The sections were treated with primary rabbit anti-GABA (SFRI-Laboratory, France) antibody diluted $1: 1500$ with phosphate buffer at $4^{\circ} \mathrm{C}$ overnight with constant agitation. The sections were then washed and treated with antirabbit IgG (biotinylated secondary antibody) for $1 \mathrm{~h}$ at room temperature. They were then processed by the $\mathrm{ABC}$ (avidine-biotin-complex) method for $1 \mathrm{~h}$ at room temperature. Finally, they were treated with $0.2 \%$ DAB containing $0.05 \% \mathrm{H}_{2} \mathrm{O}_{2}$ in Tris buffer $(\mathrm{pH}$ 7.2). The reaction was stopped by the addition of buffer, and the sections were postfixed in $1 \% \mathrm{OsO}_{4}$ for $1 \mathrm{~h}$ at $4^{\circ} \mathrm{C}$. The specimens were then dehydrated and routinely processed for electron microscopy. As controls, some sections were incubated in PBS instead of the primary antibody, and then processed in the same way (BELTZ and Burd, 1989).

We also examined the synaptic glomeruli in the superficial dorsal horn of normal mice to compare their features with those of the immuno-stained specimens.

\section{RESULTS}

Two types of synaptic glomeruli could be seen in the normal mouse superficial dosrsal horn: Type I glomeruli had centrally located CI-terminals with closely packed agranular synaptic vesicles, and were surrounded by several dendrites and axonal endings (Fig. 1a, b). Type II synaptic glomeruli had centrally located CII-terminals with evenly distributed agranular synaptic vesicles and many mitochondria, and were surrounded by dendrites and a few axonal endings containing many round or discoid synaptic vesicles (Fig. 1c, d).

Marked GABA immunoreactivity was observed in the superficial laminae of the dorsal horn (Fig. 2), but none was detected in this area of the control sections (Fig. 2). Many round or fusiform GABAergic somata were seen in the substantia gelatinosa (Fig. 3a, b). The immunoreactivity of the GABAergic interneurons was distributed throughout the neuroplasm as granular like deposits and was present on the mitochondrial membrane, but was not detected in the nucleus and nuclear envelope (Figs. 3, 6). Numerous GABAergic dendrites or axon terminals (boutons) making contact with $\mathrm{C}$-terminals were seen in the superficial dorsal horn. Large GABAergic dendrites making postsynaptic contact with CII-terminals were observed in the more superficial region (Fig. 5a-d). On the other hand, GABAergic dendrites making postsynaptic contact with CI-terminals were seen in the substantia gelatinosa (Fig. 4a, b). Small GABAergic axon terminals made symmetrical or postsynaptic contact with CI- and CII-terminals, especially the latter (Figs. 4a, 5a, c). The immunoreactivity in GABAergic dendrites and boutons was seen over the round synaptic vesicles and mitochondrial membranes. A few GABA positive soma receiving postsynaptic contact

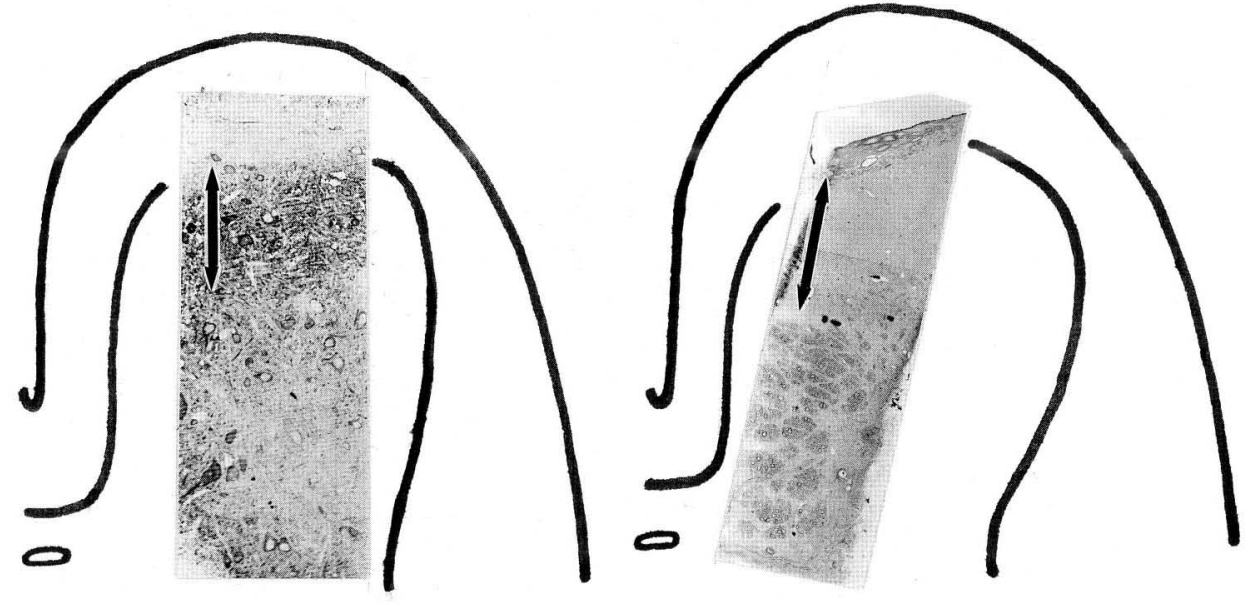

Fig. 2. Marked GABA immunoreactivity in the superficial laminae of the dorsal horn treated with anti-GABA antibody (left), and no GABA immunoreactivity in this area of a section of a control specimen (right), respectively. Arrows indicate the superficial laminae of the dorsal horn. $\times 100$ 


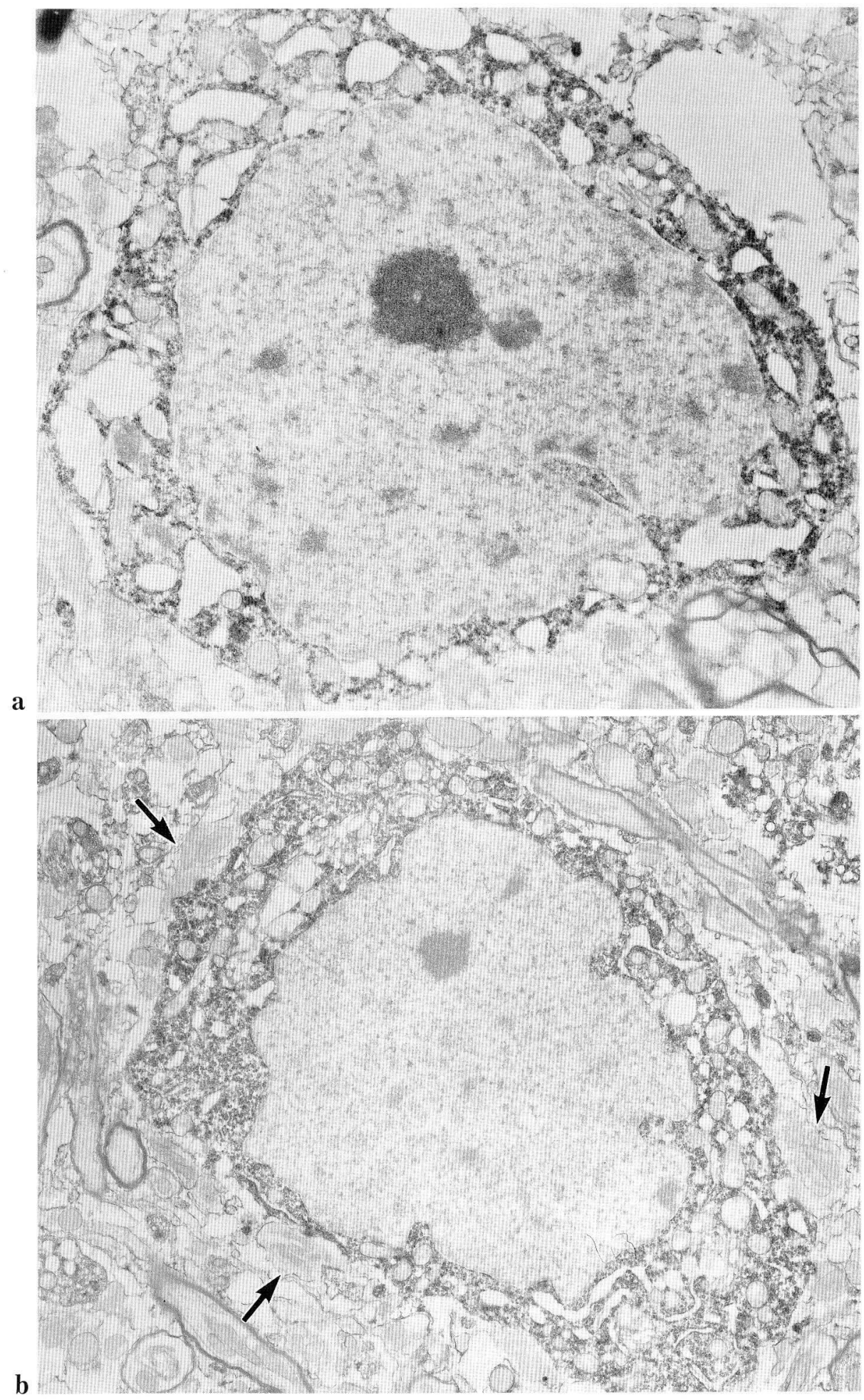

Fig. 3. GABA immunoreactive neuronal somata in the superficial dorsal horn. They are round (a) or fusiform (b). GABA immunoreactivity is seen throughout the neuroplasm and in the mitochondrial membrane, but not in the nucleus or nuclear envelope. Note the many non-GABA reactive CII-terminals contacting with the GABA positive soma (arrows). a: $\times 11,000$, b: $\times 9,000$ 


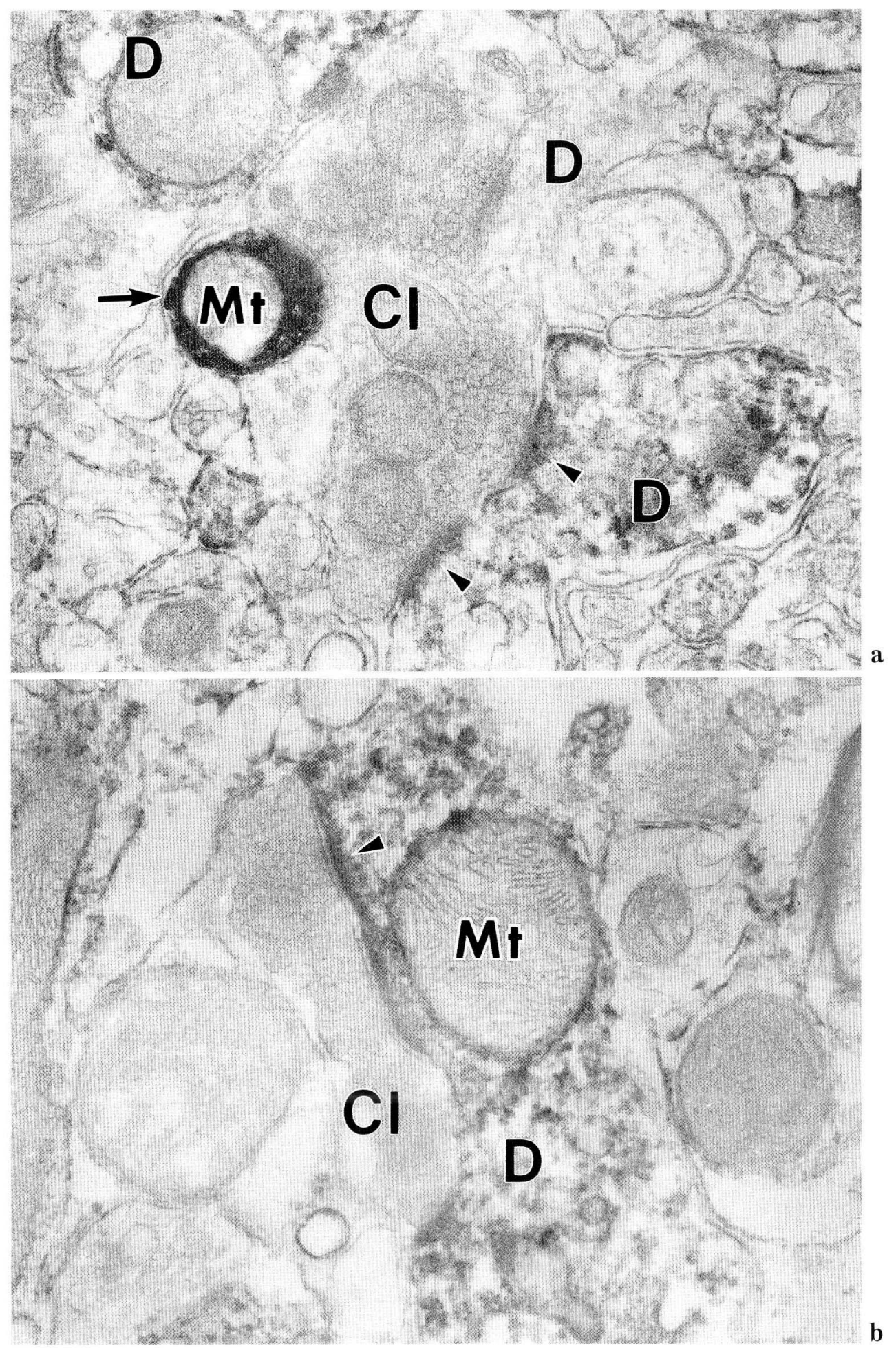

Fig. 4. GABA positive terminals showing contact with CI-terminals. a. The CI-terminal $(C I)$ make symmetrical contact with a GABA positive small axon terminal (arrow) and presynaptic contact with dendrites $(D)$. b. The CI-terminal $(C I)$ make presynaptic contact with a dendrite $(D)$. Arrowheads indicate thick, postsynaptic membranes. $M t$ mitochondria. $\times 43,000$ 
from non-reactive CII-terminals could be seen (Figs. $3 \mathrm{~b}, 6)$

Small GABAergic figures were observed to terminate near blood capillaries (Fig. 8). We identified these terminals as GABAergic neurons because of their including many GABA-positive synaptic vesicles. They were in close contact with the basal lamina of the capillary (Fig. 8a), while forming a terminal swelling (Fig. 8b). These GABAergic terminals made a rather coarse innervation around the blood capillary.

No GABA positive features could be seen in Type I or Type II synaptic glomeruli in control sections (Fig. 7).

\section{DISCUSSION}

As reported by Ribeiro-DA-Silva and CoImbra (1982), CI-terminals appeared sinuous or scallop-shaped with closely packed agranular synaptic vesicles, and few mitochondria in the electron-dense axoplasm, whereas CII-terminals were seen as large rectangular or round profiles with evenly distributed agranular synaptic vesicles, many granular synaptic vesicles and mitochondria in electron lucent axoplasm. However, as we observed previously (HIURA et al., 1990) CI-terminals are not always electron dense, even in adults. Furthermore, not the all CII-terminals

$\mathbf{a}$
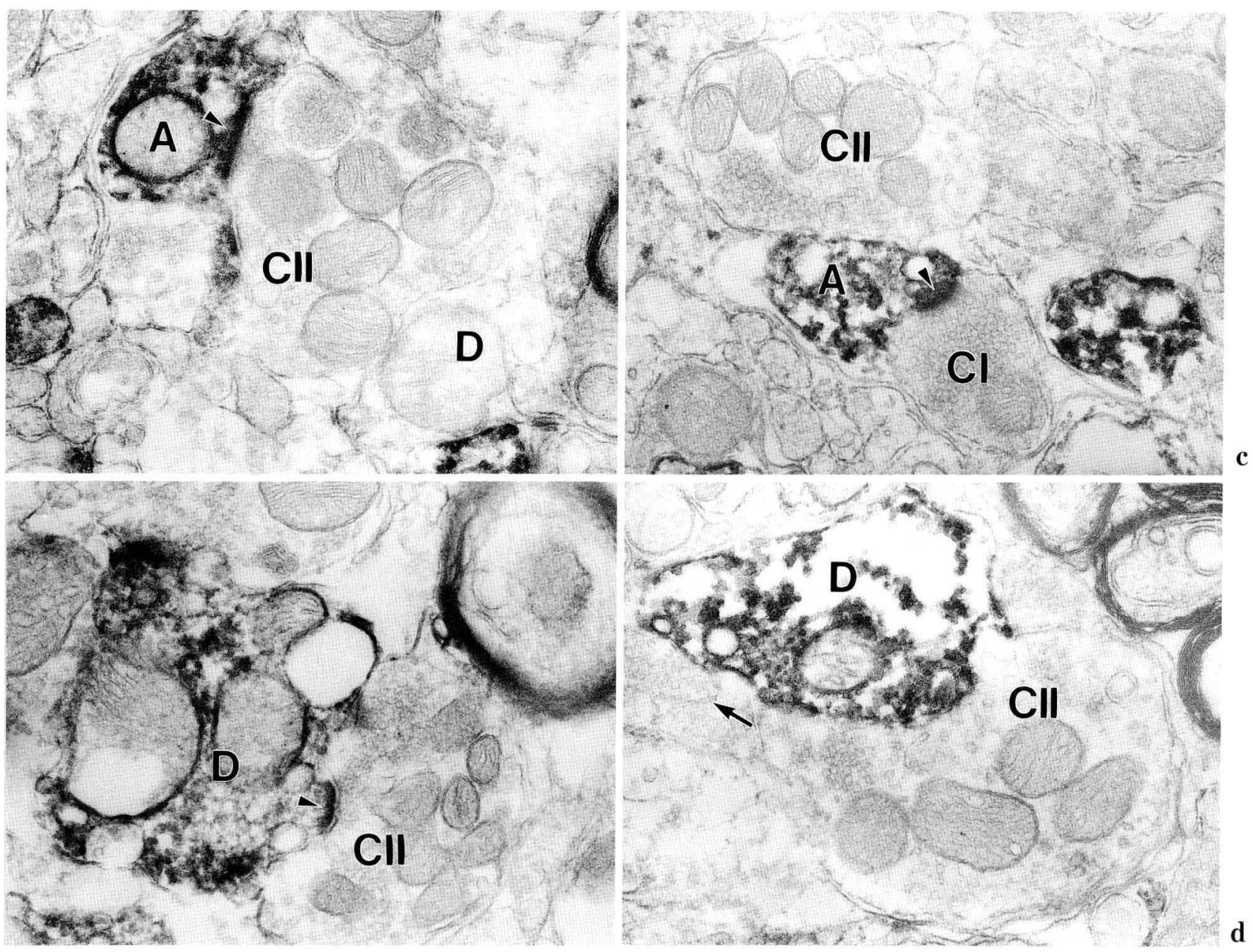

Fig. 5. GABA-positive terminals showing contact with CII-terminals $(C I I)$. a and b. CII-terminals making presynaptic contact with a small axon terminal $(A)$ and a large dendrite $(D)$. $\mathbf{c}$ and $\mathbf{d}$. CII-terminals making symmetrical contacts with a small axonal terminal $(A)$ and a large dendrite $(D)$. The GABAergic axonal terminal $(A)$ in $\mathbf{c}$ also makes postsynaptic contact with a CI-terminal $(C I)$. Note the GABA-negative axon terminal (arrow) in $\mathbf{d}$ containing many discoid vesicles. Arrowheads indicate thick, postsynaptic membranes. $\times 28,000$ 
contained many cored vesicles. The CI-terminals are mainly surrounded by several dendrites, making presynaptic contact with them, and were termed Type I synaptic glomeruli. The CII-terminals are surrounded by several dendrites and a few axonal endings including many discoid synaptic vesicles, forming presynaptic contact with the dendrites or symmetrical contact with the axonal endings, and were termed Type II synaptic glomeruli. All these features of the synaptic glomeruli in the mouse superficial dorsal horn are very similar to those reported by RibeIRo-DA-Silva and CoIMBra (1982).

GABA immunoreactivity was marked in the superficial laminae of the dorsal horn, as has been reported by many others (McLAUGHLIN et al., 1975; BARBER et al., 1978, 1982; MAGOUL et al., 1987; KADURI et al., 1987; Merighi et al., 1989; TodD and McKenzIE, 1989; CARLtON and Hayes, 1990; TODD and LOCHHEAd,

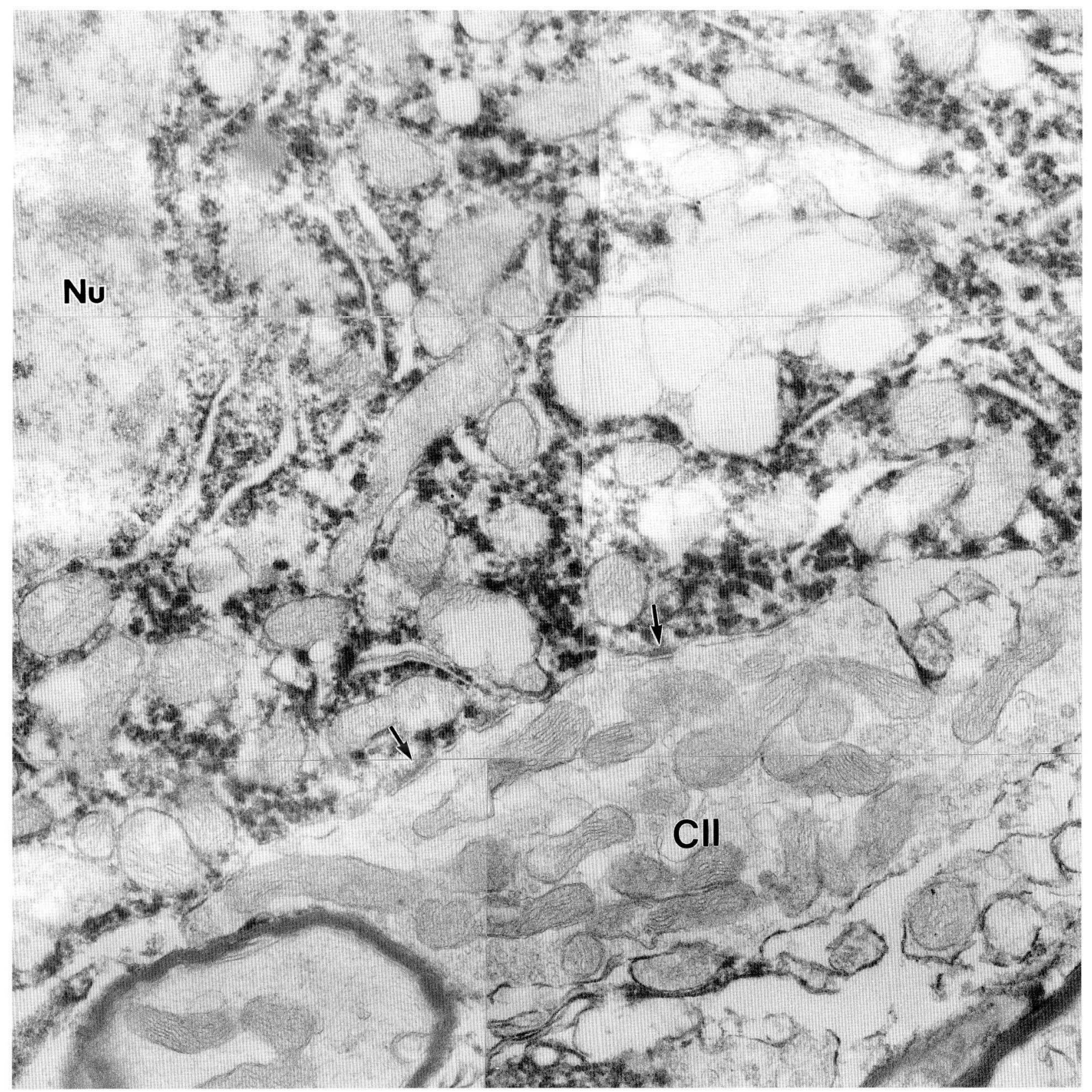

Fig. 6. A non-GABAergic CII-terminal (CII) showing presynaptic contact with the GABAergic interneuronal soma. Arrows indicate the thick, postsynaptic membrane. $N u$ nucleus. $\times 29,000$ 


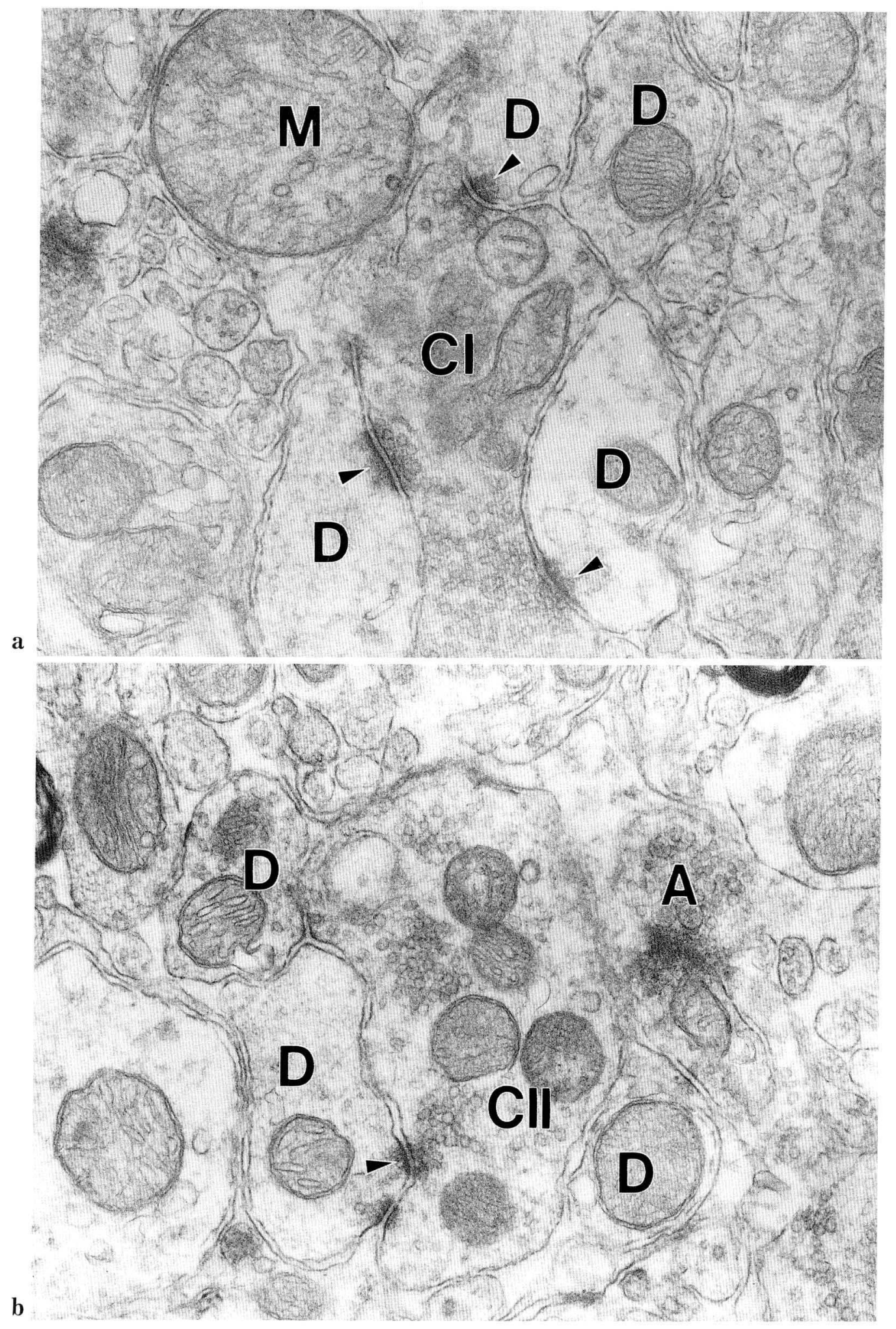

Fig. 7. Non-GABA immunoreactive Type I (a) and Type II (b) synaptic glomeruli in a control superficial dorsal horn. $C I$ a sinuous CI-terminal surrounded by dendrites $(D)$ forming postsynaptic contact with it, $C I I$ a rectangular CII-terminal surrounded by postsynaptic dendrites $(D)$, Arrowheads indicate thick postsynaptic membranes. $A$ axon terminal, $M$ mitochondria. $\times 43,000$ 


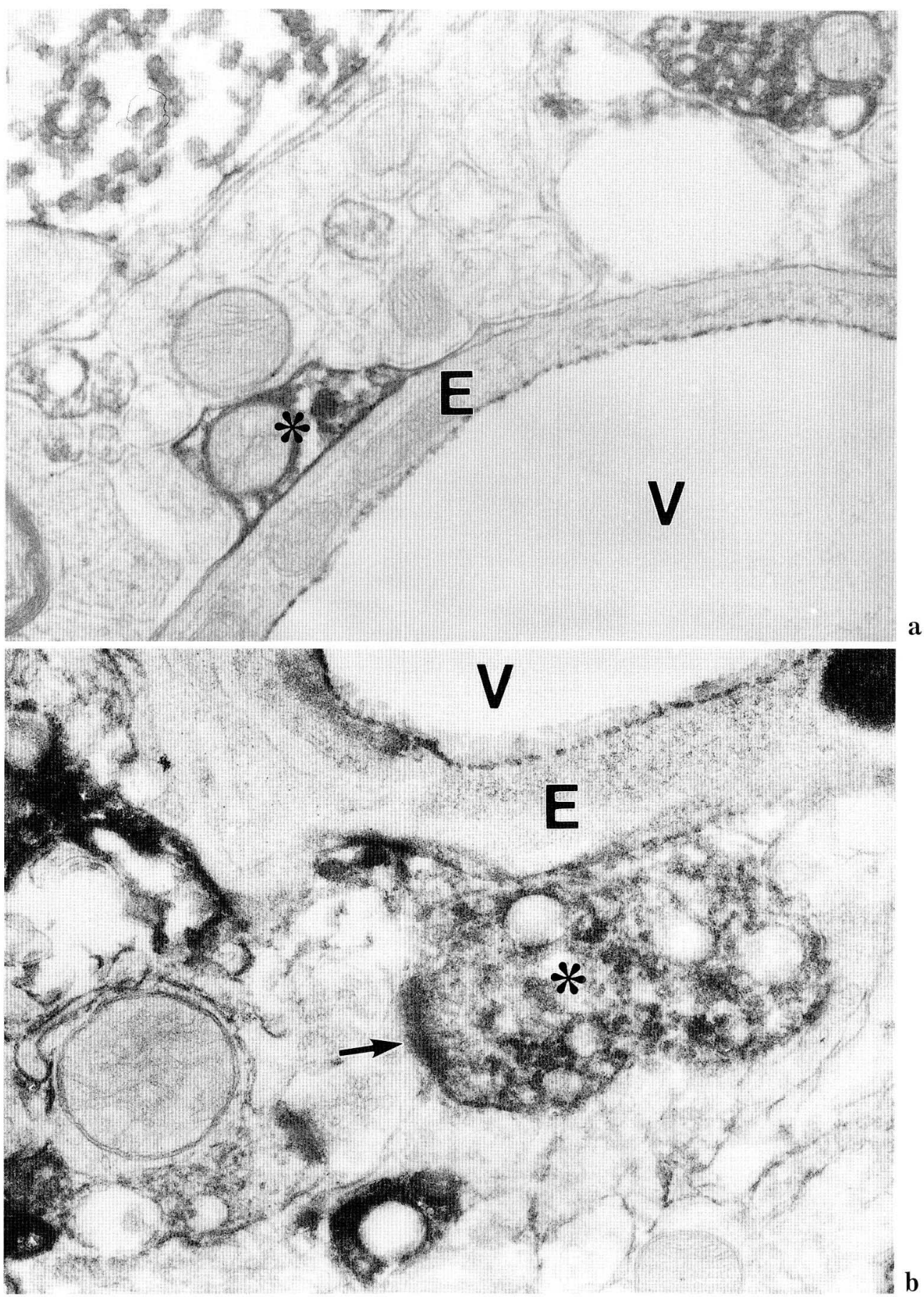

Fig. 8. GABA immunoreactive terminations (asterisks) on blood vessels (capillaries, $V$ ). a. A small terminal innervating the endothelium $(E)$. b. A small terminal innervating the endothelium $(E)$, showing a terminal swelling and synaptic contact (arrow) with axonal ending. $\times 43,000$ 
1990). Since no non-specific GABA immunoreactivity was detected in control specimens, this GABA immunoreaction is believed to be specific for neurons containing GABA. The GABA immunoreactive subcellular organellae were round synaptic vesicles, and mitochondrial membranes. These GABA-positive sites coincide with those reported by others (MCLAUGHLIN et al., 1975; BARBER et al., 1978; MAGOUL et al., 1987; KADURI et al., 1987; MERIGHI et al., 1989; CARLTON and HAYES, 1990). In particular, MERIGHI et al. clearly demonstrated GABA immunogold colloidal particles over small clear vesicles. In the GABA positive somata, immunoreactivity was distributed throughout the neuroplasm as granular deposits, but was not seen in the nucleus or its envelope. In contrast, MAGOUL et al. (1987), KADURI et al. (1987), and

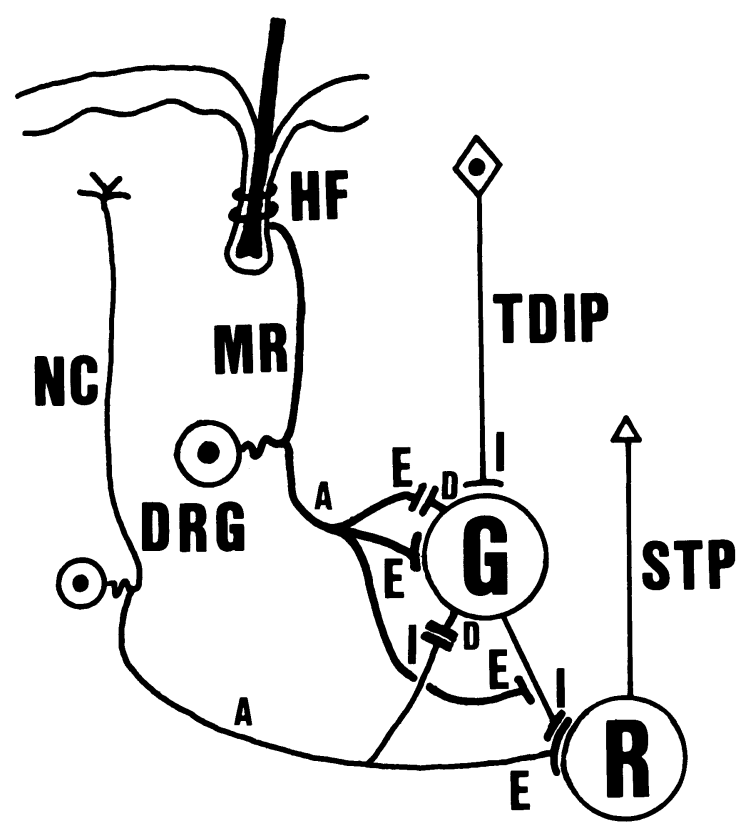

Fig. 9. Diagram indicating the modulation and relation of nociceptive information in the superficial dorsal horn. The mechano-receptive C-terminals form presynaptic contact with GABAergic interneuronal soma, its dendrites and boutons, and facilitate them. The facilitated GABAergic neuron then induces primary afferent depolarization of the nociceptive C-terminals, thereby reducing the excitability of the nociceptive relaying neurons. Thus, a decreased nociceptive impulse will be relayed to the thalamus. The position of the relaying neuron $(R)$ in the dorsal horn is now speculative. $A$ axon, $D$ dendrite, $D R G$ dorsal root ganglion neuron, $E$ excitatory, $G$ GABAergic interneuron, $H F$ hair follicle, $I$ inhibitory, $M R$ mechano-receptive fiber, $N C$ nociceptive fiber, $R$ relaying neuron, STP spinothalamic pathway, $T D I P$ tonic descending inhibitory pathway.
CARLton and Hayes (1990) observed GABA immunoreactivity in the nucleus. The reason for this difference may have been insufficient infiltration of the antibody into the tissue in our study.

Previously, GABA-positive terminals in the superficial laminae of the dorsal horn were described as presynaptic to the primary afferent central terminals, other axonal terminals, dendrites and soma (MCLAUGHLIN et al., 1975; BARBER et al., 1982; MAGOUL et al., 1987; TODD and LOCHHEAD, 1990). Recently TODD and LOCHHEAD (1990) demonstrated GABA immunoreactive dendrites, but they did not report details of the synaptic relationship between GABAergic dendrites and C-terminals. They inferred that GABA-positive boutons and dendrites indicate presynaptic axoaxonic or dendroaxonic synapses with the unmyelinated primary afferent central terminals. CARLTON and HAYES (1990) also demonstrated many GABAergic dendrites in the laminae I-III of the monkey dorsal horn. They also showed that most GABAergic dendrites are postsynaptic to the three types of primary afferent central terminals. Therefore, they proposed that neurocircuity was involved in primary afferent depolarization (PAD) by GABAergic dendrites via primary afferent facilitation. In the present study, we recognized many GABA immunoreactive dendrites making postsynaptic contact with CI- and CII-terminals. Thus, GABAergic neurons in the superficial dorsal horn of the mouse closely resemble those of the monkey. Probably, postsynaptic dendrites of GABAergic neurons have not been detected previously because the terminals are difficult to find by the degeneration method (BARBER et al., 1978) or because of the presence of dendrites containing vesicles in the dorsal horn, as pointed out by CARLTON and HAYES (1990). Two types of GABAergic features were distinguishable: large GABA positive dendrites making postsynaptic or symmetric contact with C-terminals, and small GABA-positive axon terminals making symmetrical or postsynaptic contact with CI- or CIIterminals, especially the latter. The origin of the GABAergic dendrites and boutons is unknown. TODD and MCKENZIE (1989) reported that $24-33 \%$ of the neurons in laminae I-III are GABAergic; thus, many GABAergic features may be derived from intrinsic interneurons of the superficial dorsal horn.

GABA is thought to be an inhibitory neurotransmitter in the central nervous system (CNS) (ECCLES et al., 1963; LEVy, 1977). UCHIZONO (1965) stated that terminal boutons containing discoid synaptic vesicles are inhibitory in nature. BARBER et al. (1978) reported that GABA-positive boutons include discoid vesicles, but in many studies GABA immunoreactivity was detected in terminals containing round clear vesicles 
(MCLAUGHLin et al., 1975; BARBER et al., 1978; MAGOUL et al., 1987; Merighi et al., 1989; TODD and LOCHHEAD, 1990; CARLTON and HAYES, 1990; the present observation). Thus the shape of synaptic vesicles is probably not related to the type of transmitter substance or its synaptic action (BARBER et al., 1978).

Capsaicin specifically affects nociceptive primary afferent fibers. Since capsaicin selectively destroys CI-terminals in the substantia gelatinosa, CIterminals are considered to be the central terminals of nociceptive primary afferents (RIBEIRO-DA-SILVA and CoIMBRA, 1984). On the other hand, CII-terminals without neurofilaments are considered to be central terminals of small myelinated fibers showing little degeneration after capsaicin treatment (RIBEIRO-DASilva and CoIMBRA, 1982, 1984). It has been well established that thin myelinated and unmyelinated fibers mainly terminate in the superficial dorsal horn as shown in anatomical (LIGHT and PERL, 1977; RIBEIRO-DA-SILVA and CoIMBRA, 1982) and physiological (Light et al., 1979) studies. Moreover, LighT et al. (1979) demonstrated that myelinated and C-fibers of cutaneous innocuous mechanoreceptors predominantly terminate in the substantia gelatinosa. Most of the fine myelinated fiber axons originate from hair receptors and carry more than one type of sensory information (BuRGess and PerL, 1967). A study on iontophoretic injection of HRP into hair follicle afferent fibers with immunocytochemical staining of the $\gamma$ aminobutyric acid decarboxylase $(\mathrm{GAD})$, responsible for synthesizing GABA, revealed that hair follicle afferent central terminals in the dorsal horn make postsynaptic contact with GAD-positive boutons (MAXELl and NoBle, 1987). However, the morphological characterization of pre- and postsynaptic terminals and the relationship between them was not clear in that study. Furthermore, it is known that nociceptive information is modulated by primary afferent fibers in the superficial dorsal horn (CERVERO, 1986; Steedman and Molony, 1987; SteEdman, 1989). From these previous findings and the present study, we propose that C-terminals of mechanoreceptive primary afferents facilitate the GABAergic neurons, and result in primary afferent deporalization (PAD) of nociceptive primary afferent fibers (Fig. 9). Consequently, closing the gate in the superficial dorsal horn as proposed by MELZACK and WALL (1965) and WALL (1978) appears to result in analgesia. These findings seem to demonstrate the anatomical basis for the gate control theory that nociceptive information is modulated by primary afferent fibers in the spinal dorsal horn.

Some small terminals including GABA-positive synaptic vesicles terminated on blood capillaries. So far as we know, GABA has not been considered to have a vasomotor function. BARBER et al. (1982) demonstrated GABAergic cerebrospinal-contacting neurons among the rat spinal cord ependymal cells surrounding the central canal. They suggested a neuroendocrine modulation of these neurons in the spinal cord. It is believed that the GABAergic neurons innervating the blood capillaries demonstrated in the present study seem to be neurosecretory rather than vasomotor in nature. The functional significance of GABA presumably conveyed via blood circulation remains to be determined.

This is the first demonstration of an anatomical basis for the gate control theory and of the GABAergic innervation of the blood capillary.

\section{REFERENCES}

Barber, R. P., J. E. Vaughn, K. Saito, B. J. McLaughlin and E. RoBERTs: GABAergic terminals are presynaptic to primary afferent terminals in the substantia gelatinosa of the rat spinal cord. Brain. Res. 141: 35-55 (1978).

Barber, R. P., J. E. VAughn and E. Roberts: The cytoarchitecture of GABAergic neurons in rat spinal cord. Brain Res. 238: 305-328 (1982).

Beltz, B. S. and G. D. Burd: Specificity in immunocytochemistry. In: (ed. by) B. S. BELTZ and G. D. BuRD: Immunocytochemical technique: principles and practice. Blackwell Scientific Publication, Cambridge, 1989 (p. 29).

Burgess, P. R. and E. R. Perl: Myelinated afferent fibers responding specifically to noxious stimulation of the skin. J. Physiol. 190: 541-562 (1967).

Carlton, S. M. and E. S. Hayes: Light microscopic and ultrastructural analysis of GABA-immunoreactive profiles in the monkey spinal cord. J. Comp. Neurol. 300: 162-182 (1990).

Cervero, F.: Dorsal horn neurons and their sensory inputs. In: (ed. by) T. L. YAKSH: Spinal afferent processing. Plenum Press, New York, 1986 (p. 197-216).

-: The superficial dorsal horn and pain. In: (ed. by) F. Cervero, G. J. Benett and P. M. Headly: Processing of sensory information in the superficial dorsal horn of the spinal cord. Plenum Press, New York, 1989 (p. 5).

Eccles, J. C., R. SchmidT and W. D. Willis: Pharmacological studies on presynaptic inhibition. J. Physiol. 168: 500-530 (1963).

Hiura, A., E. L. Villalobos and H. Ishizuka: The action of capsaicin on primary afferent central terminals in the superficial dorsal horn of newborn mice. Arch. Histol. Cytol. 53: 455-466 (1990).

Kaduri, A. J., R. Magoul, L. Lescaudron, G. CampisTRON and A. Calas: Immunocytochemical approach of 
GABAergic innervation of the mouse spinal cord using antibodies to GABA. J. Hirnforsch. 28: 349-355 (1987).

LEVy, R. A.: The role of GABA in primary afferent depolarization. Prog. Neurobiol. 9: 211-267 (1977).

Light, A. R. and E. R. PerL: Differential termination of large-diameter and small-diameter primary afferent fibers in the spinal dorsal gray matter as indicated by labeling with horseradish peroxidase. Neurosci. Lett. 6: 59-63 (1977)

Light, A. R., D. L. Tervino and E. R. Perl: Morphological features of functionally defined neurons in the marginal zone and substantia gelatinosa of the spinal dorsal horn. J. Comp. Neurol. 186: 151-172 (1979).

Magoul, R., B. Onteniente, M. Geffard and A. Calas: Anatomical distribution and ultrastructural organization of the GABAergic system in the rat spinal cord: an immunocytochemical study using anti-GABA antibodies. Neuroscience 20: 1001-1009 (1987).

Maxell, D. J. and R. Noble: Relationships between hair-follicle afferent terminations and glutamic acid decarboxylase containing boutons in the cat's spinal cord. Brain Res. 408: 308-312 (1987).

Mclaughlin, B. J., R. Barber, K. Saito, E. Roberts and J. Y. WU: Immunocytochemical localization of glutamate decarboxylase in rat spinal cord. J. Comp. Neurol. 164: 305-322 (1975).

Merighi, A., J. M. Polak, G. Fumagalli and D. T. Theodosis: Ultrastructural localization of neuropeptides and GABA in rat dorsal horn: a comparison of different immunogold labeling techniques. J. Histochem. Cytochem. 37: 529-540 (1989).

Melzack, R. and P. D. Wall: Pain mechanism: a new theory - a gate control system modulates sensory input from the skin before it evokes pain perception and response. Science 19: 971-979 (1965).

Ribeiro-Da-Silva, A. and A. CoImbra: Two types of synaptic glomeruli and their distribution in laminae IIII of the rat spinal cord. J. Comp. Neurol. 209: 176-186 (1982).

- Capsaicin causes selective damage to type I synaptic glomeruli in rat substantia gelatinosa. Brain Res. 290: 380-383 (1984).
Steedman, W. M.: The influence of cutaneous inputs on the activity of neurons in the substantia gelatinosa. In: (ed. by) F. Cervero, G. J. Benett and P. M. Headly: Processing of sensory information in the superficial dorsal horn of the spinal cord. Plenum Press, New York, 1989 (p. 145-158).

Steedman, W. M. and V. Molony: Intracellularly recorded responses of neurons in laminae II of cat spinal dorsal horn to activation of cutaneous afferent inputs. In: (ed. by) R. F. Schmidt, H.-G. Schaible and C. VAHLE-HINZ: Fine afferent nerve fibers and pain. VCH Publishers, Weinheim, 1987 (p. 289-297).

TodD, A. J. and V. LochHead: GABA-like immunoreactivity in type I glomeruli of rat substantia gelatinosa. Brain Res. 514: 171-174 (1990).

TodD, A. J. and J. McKenzie: GABA-immunoreactive neurons in the dorsal horn of the rat spinal cord. Neuroscience 31: 799-806 (1989).

Uchizono, K.: Characteristics of excitatory and inhibitory synapses in the central nervous system of the cat. Nature 207: 642-643 (1965).

W ALL, P. D.: The gate control theory of pain mechanisms: a re-examination and re-statement. Brain, 101: 1-18 (1978) 